\title{
ULTRA-SMALL SCALE-FREE GEOMETRIC NETWORKS
}

\author{
J. E. YUKICH, ${ }^{*}$ Lehigh University
}

\begin{abstract}
We consider a family of long-range percolation models $\left(G_{p}\right)_{p>0}$ on $\mathbb{Z}^{d}$ that allow dependence between edges and have the following connectivity properties for $p \in$ $(1 / d, \infty)$ : (i) the degree distribution of vertices in $G_{p}$ has a power-law distribution; (ii) the graph distance between points $\boldsymbol{x}$ and $\boldsymbol{y}$ is bounded by a multiple of $\log _{p d} \log _{p d}|\boldsymbol{x}-\boldsymbol{y}|$ with probability $1-o(1)$; and (iii) an adversary can delete a relatively small number of nodes from $G_{p}\left(\mathbb{Z}^{d} \cap[0, n]^{d}\right)$, resulting in two large, disconnected subgraphs.
\end{abstract}

Keywords: Scale-free graph; long-range percolation; chemical distance

2000 Mathematics Subject Classification: Primary 60D05

Secondary $05 \mathrm{C} 80$

\section{Introduction}

The statistical properties of large networks have received considerable attention in the recent scientific literature [2], [14], [21], [25]. Of special interest are the power-law random networks in which the fraction of vertices of degree $k$ is proportional to $k^{-q}$ for some $q>0$. Such networks lack an inherent scale and have been termed 'scale free'. Scale-free graphs are ubiquitous in random network theory and have been proposed as a way to model the behavior of technological, social, and biological networks [1], [21].

Networks often have a geometric component to them where the vertices have positions in space and geographic proximity plays a role in deciding which vertices get connected. In this context, random geometric graphs are a natural alternative to the classical Erdôs-Rényi random graph models. Random connection models [20] provide one way to describe networks with spatial content. In these models the event, $E_{\boldsymbol{x}, \boldsymbol{y}}$, of a connection between points $\boldsymbol{x}$ and $\boldsymbol{y}$ has probability $p_{\boldsymbol{x}, \boldsymbol{y}}:=\mathrm{P}\left[E_{\boldsymbol{x}, \boldsymbol{y}}\right]=g(|\boldsymbol{x}-\boldsymbol{y}|)$, where $g: \mathbb{R}^{+} \rightarrow[0,1]$ is a connection function and $|\boldsymbol{x}|$ denotes the Euclidean norm of $\boldsymbol{x}$. The standard long-range percolation model assumes independence of $E_{\boldsymbol{x}, \boldsymbol{y}}$ and $E_{\boldsymbol{x}, \boldsymbol{u}}, \boldsymbol{y} \neq \boldsymbol{u}$, which may not be the case in networked systems. Moreover, the degree distribution in this connection model generally does not follow a power law.

Allowing dependency between edges will in general result in technically more complicated models. In this note we show that a natural edge dependency gives rise to a family of longrange percolation models, $\left(G_{p}\right)_{p>0}$, which is technically tractable and which exhibits three connectivity properties for $p \in(1 / d, \infty)$. First, $G_{p}$ has a power-law distribution. Second, $G_{p}$ is ultra-small, in the sense that the graph distance between lattice points $\boldsymbol{x}$ and $\boldsymbol{y}$ is bounded by a multiple of $\log _{p d} \log _{p d}|\boldsymbol{x}-\boldsymbol{y}|$ with probability $1-o(1)$, where $o(1)$ denotes a quantity tending to 0 as $|\boldsymbol{x}-\boldsymbol{y}| \rightarrow \infty$. Ultra-small graph distances imply efficiency, are consistent

Received 13 April 2005; revision received 3 May 2006.

* Postal address: Department of Mathematics, Lehigh University, Bethlehem, PA 18015, USA.

Email address: joseph.yukich@lehigh.edu

Partially supported by NSF grant DMS-0203720. 
with the 'small-world phenomenon' [2], [14], [24], [25], and are relevant in the context of routeing, searching, and transport of information. Third, an adversary can delete a relatively small number of nodes from $G_{p}\left(\mathbb{Z}^{d} \cap[0, n]^{d}\right)$, after which there are two disconnected subgraphs, each containing nearly one-half of the total number of network nodes.

\subsection{A general dependent random connection model}

Let $\left\{U_{z}\right\}_{z \in \mathbb{Z}^{d}}$ be independent, identically distributed uniform $[0,1]$ random variables indexed by $\mathbb{Z}^{d}$. Let $p>0$ and $\delta \in(0,1]$. For each $z \in \mathbb{Z}^{d}$, we take $\delta U_{z}^{-p}$ to represent a weight at node $z$ defining the radius of the 'ball of influence' at $z$. Consider the graph $G_{p, \delta}:=G_{p, \delta}\left(\mathbb{Z}^{d}\right)$ which puts an edge between nodes $\boldsymbol{x}, \boldsymbol{y} \in \mathbb{Z}^{d}$ whenever each node is contained in the other's ball of influence. Thus, this connection rule says that the edge $(\boldsymbol{x}, \boldsymbol{y})$ appears in $G_{p, \delta}\left(\mathbb{Z}^{d}\right)$ whenever

$$
|\boldsymbol{x}-\boldsymbol{y}| \leq \delta \min \left(U_{\boldsymbol{x}}^{-p}, U_{\boldsymbol{y}}^{-p}\right) .
$$

Let $\delta=1$. By the independence of the $U_{z}$, we have $p_{\boldsymbol{x}, \boldsymbol{y}}:=\mathrm{P}\left[E_{\boldsymbol{x}, \boldsymbol{y}}\right]=|\boldsymbol{x}-\boldsymbol{y}|^{-2 / p}$, showing that the probability of (there being) long edges in $G_{p}:=G_{p, 1}$ increases with $p$. Edges in $G_{p}$ have dependent probabilities: if $|\boldsymbol{y}|<|\boldsymbol{x}|$ then the probability of the edge $(\mathbf{0}, \boldsymbol{y})$ given the edge $(\boldsymbol{0}, \boldsymbol{x})$ is $|\boldsymbol{y}|^{-1 / p}$ instead of $|\boldsymbol{y}|^{-2 / p}$.

The family of random connection models $G_{p, \delta}$ is disconnected for general $p$ and $\delta$, but not for $\delta=1$, since having $U_{z}^{-p} \geq 1$ for all $z \in \mathbb{Z}^{d}$ implies that adjacent lattice points are connected in $G_{p}$. The main results below show, for all $p \in(1 / d, \infty)$, that the components of $G_{p}$ are of arbitrarily large diameter with arbitrarily large probability. Moreover, in accordance with their Poisson Boolean model counterparts (cf. [20]), it is easy to check, for all $\delta \in(0,1]$ and large $p$, that the expected number of nodes in the component of $G_{p, \delta}$ containing $\mathbf{0}$ is infinite, whereas, for $p$ and $\delta$ both small, the expected number of such nodes is finite. Our purpose here is to explore the connectivity properties of $G_{p}, p \in(1 / d, \infty)$.

\subsection{Main results}

Let $D_{p}(\mathbf{0})$ denote the degree of the origin in $G_{p}\left(\mathbb{Z}^{d}\right)$, let $\omega_{d}$ denote the volume of the unitradius ball in $\mathbb{R}^{d}$, and let $\alpha:=p d-1$. Our first result shows that if $p \in(1 / d, \infty)$ then the degree of a typical vertex follows a power law, i.e. $G_{p}$ is scale free.

Theorem 1.1. $\left(G_{p}\left(\mathbb{Z}^{d}\right)\right.$ has a power-law degree distribution.) For all $d=1,2, \ldots$ and all $p \in(1 / d, \infty)$,

$$
\lim _{t \rightarrow \infty} t^{1 / \alpha} \mathrm{P}\left[D_{p}(\mathbf{0})>t\right]=\left(p d \omega_{d} / \alpha\right)^{1 / \alpha} .
$$

For all $\boldsymbol{x}, \boldsymbol{y} \in \mathbb{Z}^{d}, d_{p}(\boldsymbol{x}, \boldsymbol{y})$ denotes the $G_{p}$ graph distance ('chemical distance') between $\boldsymbol{x}$ and $\boldsymbol{y}$. Our next result says that $G_{p}$ is ultra-small (cf. [12]), in that $d_{p}(\boldsymbol{x}, \boldsymbol{y})$ is bounded by $4(2+\log \log |\boldsymbol{x}-\boldsymbol{y}|)$ with probability $1-o(1)$, where throughout, for all $s>0, \log s$ is short for $\log _{p d} s$. We expect that the upper bound in this result can be improved but have not tried to obtain the sharpest bound.

Theorem 1.2. $\left(G_{p}\left(\mathbb{Z}^{d}\right)\right.$ has small graph distance.) For all $d=1,2, \ldots$ and all $p \in(1 / d, \infty)$,

$$
\frac{d_{p}(\mathbf{0}, \boldsymbol{x})}{2+\log \log |\boldsymbol{x}|} \leq 4
$$

with probability $1-o(1)$, where o(1) tends to 0 as $|\boldsymbol{x}| \rightarrow \infty$. 
The network failure of $G_{p}\left(\mathbb{Z}^{d}\right)$ is easily quantified, as follows.

Theorem 1.3. (Network failure.) For all $d=1,2, \ldots$ and all $p \in(1 / d, \infty)$, an adversary can delete $N$ nodes from $G_{p}\left(\mathbb{Z}^{d} \cap[0, n]^{d}\right)$, where $\mathrm{E}[N]=O\left(n^{d-1}\left[n^{1-1 / p} \vee 1\right]\right)$, resulting in two disconnected subgraphs on vertex sets of cardinality at least $n^{d} / 2-N$.

Theorem 1.3 implies, in particular, that if $p \in(1 / d, 1)$ then removing roughly $O\left(n^{d-1}\right)$ nodes may reduce $G_{p}\left(\mathbb{Z}^{d} \cap[0, n]^{d}\right)$ to two large, disconnected subgraphs.

Remarks. 1. Standard long-range percolation models. Assume that $p_{\boldsymbol{x}, \boldsymbol{y}}:=\mathrm{P}\left[E_{\boldsymbol{x}, \boldsymbol{y}}\right]=$ $|\boldsymbol{x}-\boldsymbol{y}|^{-s+o(1)}$ as $|\boldsymbol{x}-\boldsymbol{y}| \rightarrow \infty$, for some constant $s \in(0, \infty) ; E_{\boldsymbol{x}, \boldsymbol{y}}$ and $E_{\boldsymbol{x}, \boldsymbol{u}}$ are independent for all $\boldsymbol{x}, \boldsymbol{y}, \boldsymbol{u} \in \mathbb{Z}^{d}$. For $s \in(0, d)$, Benjamini et al. [4] showed that the graph distance $d(\mathbf{0}, \boldsymbol{x})$ behaves like the constant $\lceil s /(d-s)\rceil$ as $|\boldsymbol{x}| \rightarrow \infty$. Here, $\lceil x\rceil$ denotes the greatest integer less than $x$. For $s=d$, Coppersmith et al. [13] showed that $d(\mathbf{0}, \boldsymbol{x})$ scales as $\log |\boldsymbol{x}| / \log \log |\boldsymbol{x}|$, whereas, for $s \in(d, 2 d)$, Biskup [7], [8] showed that $d(\mathbf{0}, \boldsymbol{x})$ scales as $(\log |\boldsymbol{x}|)^{\Delta+o(1)}$, where $\Delta:=\Delta(s, d):=\log 2 / \log (2 d / s)$. The case $s=2 d$ is open and, for $s \in(2 d, \infty), d(\mathbf{0}, \boldsymbol{x})$ scales at least linearly in $|\boldsymbol{x}|$, as shown by Berger [5]. The different scalings for the standard long-range percolation model suggest that $G_{p}$ also has different scalings for $p \in(0,1 / d)$, but we have not determined them. Kleinberg [19] proposed a lattice model where long-range contacts are added in a biased way, there being, however, a uniform bound on the number of such contacts.

2. Geometric networks in $\mathbb{R}^{d}$. We expect that Theorems 1.1-1.3 extend to analogously defined continuum models on Poisson point sets in $\mathbb{R}^{d}$. This would add to the following related results.

(a) Let $f: \mathbb{R}^{d} \rightarrow \mathbb{R}^{+}$and let $\mathcal{P}_{f}$ be a Poisson point process on $\mathbb{R}^{d}$ with intensity $f$. The geometric graph, described in depth by Penrose [23], joins two nodes in $\mathcal{P}_{f}$ whenever their Euclidean distance is less than a specified cutoff. Hermann et al. [18, Section II.B] showed that if $\int_{\mathbb{R}^{d}} f^{r}(\boldsymbol{x}) \mathrm{d} \boldsymbol{x}=\infty$ for all $r>r_{0}$, then the degree distribution is effectively a power law.

(b) The on-line nearest-neighbors graph is defined on randomly ordered point sets in $\mathbb{R}^{d}$, and places an edge between each point and its nearest neighbor amongst the points preceding it in the ordering. Such graphs have scale-free properties over certain degree domains [6], [16].

(c) Franceschetti and Meester [17] developed a scale-free continuum model but did not obtain iterated log bounds on interpoint graph distances.

(d) The standard Boolean connection model puts an edge between $\boldsymbol{x}$ and $\boldsymbol{y}$ whenever the respective balls of influence overlap. In the context of $(1.1),(\boldsymbol{x}, \boldsymbol{y})$ is an edge whenever $|\boldsymbol{x}-\boldsymbol{y}| \leq \delta\left(U_{\boldsymbol{x}}^{-p}+U_{\boldsymbol{y}}^{-p}\right)$. These models are not in general scale free.

3. Power exponents $q \in(2,3)$. Consider a random graph on $n$ nodes $\boldsymbol{v}_{1}, \boldsymbol{v}_{2}, \ldots, \boldsymbol{v}_{n}$ with weight (expected degree) $w_{i}$ at node $\boldsymbol{v}_{i}$. Nodes $\boldsymbol{v}_{i}$ and $\boldsymbol{v}_{j}$ are connected with probability $\rho w_{i} w_{j}$, where $\rho=\left(\sum_{i=1}^{n} w_{i}\right)^{-1}$. Chung and Lu [10], [11] provided conditions on the weights under which the degree distribution is proportional to $k^{-q}, q \in(2,3), k \in \mathbb{Z}$, the average distance between nodes is almost surely $O(\log \log n)$, and the diameter is $O(\log n)$. In unrelated work, Cohen and Havlin [12] argued that whenever the degree distribution of a random graph on $n$ vertices is proportional to $k^{-q}$, where $q \in(2,3), k$ is restricted to $(m, K)$, and where $m$ and $K:=K(n)$ are well-defined 'cutoffs', then the diameter behaves like $\log \log n$. 
4. Preferential attachment models. These dynamic graphs evolve with time in such a way that a newly arriving vertex connects to an existing vertex with a probability proportional to the degree of the (latter) vertex. Thus, nodes of high degree tend to acquire more new links than do nodes of low degree. Albert and Barabási [1] showed that such models follow a power law, are not geometry dependent, and, as shown by Bollabás and Riordan [9], are not ultra-small in general.

5. Degree dependence on $p$. Theorem 1.1 tells us that $\mathrm{P}\left[D_{p}(\mathbf{0})=k\right] \sim C k^{-q}$, where $q:=p d /(p d-1)$. Thus, as $p$ increases on $(1 / d, \infty)$, the exponent of the degree distribution, $q$, decreases to 1 .

6. Further connectivity results. Theorems 1.1-1.3 describe the connectivity of $G_{p}\left(\mathbb{Z}^{d}\right)$. Further analysis of the connectivity of $G_{p}\left(\mathbb{Z}^{d}\right)$, such as thermodynamic and Gaussian limits for the number of three cycles (or other clustering coefficients) on $G_{p}\left(\mathbb{Z}^{d} \cap[0, n]^{d}\right.$ ), is simplified by appealing to the stabilization properties of $G_{p}$ (see especially [22]). $G_{p}\left(\mathbb{Z}^{d}\right)$ is assortative in that high-degree nodes tend to link to high-degree nodes and low-degree nodes tend to link to low-degree nodes.

7. The case $p \in(0,1 / d)$. If $p \in(0,1 / d)$ then $G_{p}$ has few long edges and the proofs of the scale-free and ultra-small properties break down. The scalar $1 / d$ thus represents the boundary between graphs that are ultra-small scale free and those which are not.

\section{Proof of Theorem 1.1}

Throughout, we adopt the following notation: $B_{r}(\boldsymbol{x})$ denotes the Euclidean ball of radius $r$ centered at $\boldsymbol{x} \in \mathbb{R}^{d}, L_{r}(\boldsymbol{x}):=B_{r}(\boldsymbol{x}) \cap \mathbb{Z}^{d} \backslash\{\boldsymbol{x}\}$ denotes the lattice points a distant at most $r$ from $\boldsymbol{x}$, and $C$ denotes a generic positive constant whose value may change from line to line. The underlying probability space is $\Omega:=[0,1]^{\mathbb{Z}^{d}}$ and is equipped with the product probability measure $\mathrm{P}:=\mu^{\mathbb{Z}^{d}}$, where $\mu$ is the uniform probability measure on $[0,1]$. Conditional on $U_{\mathbf{0}}=u, D_{p}(\mathbf{0})$ is the number of points $\boldsymbol{y}$ in $L_{u^{-p}}(\mathbf{0})$ with weight, $U_{\boldsymbol{y}}^{-p}$, exceeding $|\boldsymbol{y}|$; hence, $U_{\boldsymbol{y}} \in\left[0,|\boldsymbol{y}|^{-1 / p}\right]$. Writing $D\left(u^{-p}\right)$ for the value of $D_{p}(\mathbf{0})$ conditioned on $\mathbf{0}$ having weight $u^{-p}$, we have

$$
D\left(u^{-p}\right)=\sum_{\boldsymbol{y} \in L_{u^{-}}(\mathbf{0})} 1_{\left\{U_{\boldsymbol{y}} \leq|\boldsymbol{y}|^{-1 / p}\right\}} .
$$

Thus, to prove Theorem 1.1 we condition on $U_{\mathbf{0}}$ and show that

$$
\lim _{t \rightarrow \infty} t^{1 / \alpha} \int_{0}^{1} \mathrm{P}\left[D\left(u^{-p}\right)>t\right] \mathrm{d} u=\left(\frac{p d \omega_{d}}{\alpha}\right)^{1 / \alpha},
$$

where, recall, $\alpha:=p d-1$. The next lemma will be useful in establishing (2.1). Let $\beta:=$ $p d \omega_{d} / \alpha$.

Lemma 2.1. For all $p \in(1 / d, \infty)$, we have

$$
\mathrm{E}\left[D\left(u^{-p}\right)\right]=\beta u^{-\alpha}+O\left(\max \left(1, u^{-p d+p+1}\right)\right),
$$

where the error on the right-hand side of (2.2) holds as $u \rightarrow 0^{+}$.

Proof. Note that $\mathrm{E}\left[D\left(u^{-p}\right)\right]$ is approximated by

$$
\int_{|\boldsymbol{x}| \leq u^{-p}}|\boldsymbol{x}|^{-1 / p} \mathrm{~d} \boldsymbol{x}=d \omega_{d} \int_{0}^{u^{-p}} t^{d-1-1 / p} \mathrm{~d} t=\beta u^{-\alpha} .
$$


Let $R:=R(u)$ be the maximal collection of grid cubes (cubes centered at points in $\mathbb{Z}^{d}$ with edge length 1) contained within $B_{u^{-p}}(\mathbf{0})$. The approximation error

$$
\left.\left|\mathrm{E}\left[D\left(u^{-p}\right)\right]-\int_{|\boldsymbol{x}| \leq u^{-p}}\right| \boldsymbol{x}\right|^{-1 / p} \mathrm{~d} \boldsymbol{x} \mid
$$

is bounded by the sum of the following three errors:

$$
\begin{aligned}
& E_{1}:=\left.\left|\mathrm{E}\left[D\left(u^{-p}\right)\right]-\sum_{\boldsymbol{y} \in R(u) \cap \mathbb{Z}^{d}, \boldsymbol{y} \neq \mathbf{0}}\right| \boldsymbol{y}\right|^{-1 / p} \mid, \\
& E_{2}:=\left.\left|\sum_{\boldsymbol{y} \in R(u) \cap \mathbb{Z}^{d}, \boldsymbol{y} \neq \mathbf{0}}\right| \boldsymbol{y}\right|^{-1 / p}-\int_{R(u)}|\boldsymbol{x}|^{-1 / p} \mathrm{~d} \boldsymbol{x} \mid, \\
& E_{3}:=\left.\left|\int_{R(u)}\right| \boldsymbol{x}\right|^{-1 / p} \mathrm{~d} \boldsymbol{x}-\int_{|\boldsymbol{x}| \leq u^{-p}}|\boldsymbol{x}|^{-1 / p} \mathrm{~d} \boldsymbol{x} \mid .
\end{aligned}
$$

Now,

$$
E_{1}=\sum_{\boldsymbol{y} \in\left(B_{u^{-}-p(\mathbf{0})} \backslash R(u)\right) \cap \mathbb{Z}^{d}, \boldsymbol{y} \neq \mathbf{0}}|\boldsymbol{y}|^{-1 / p}
$$

and, so, is bounded by the product of

$$
\operatorname{card}\left\{\left(B_{u^{-p}}(\mathbf{0}) \backslash R(u)\right) \cap \mathbb{Z}^{d}\right\} \quad \text { and } \quad \sup \left\{y \in\left(B_{u^{-p}}(\mathbf{0}) \backslash R(u)\right) \cap \mathbb{Z}^{d}:|y|^{-1 / p}\right\} .
$$

Since the first factor is bounded by $C u^{-p(d-1)}$ and the second by $C u$, it follows that $E_{1} \leq$ $C u^{-p d+p+1}$. A similar method shows that $E_{3} \leq C u^{-p d+p+1}$.

We estimate $E_{2}$ as follows. For all $\boldsymbol{y} \in \mathbb{Z}^{d}$, let $Q_{\boldsymbol{y}}$ denote the grid cube with center $\boldsymbol{y}$. For all $s=1,2, \ldots$, let $M(s):=\operatorname{card}\left\{y \in \mathbb{Z}^{d}:|\boldsymbol{y}| \in[s, s+1)\right\}$. Since there is a constant $C>0$ such that, for all $\boldsymbol{x} \in Q_{\boldsymbol{y}}$ and all $\boldsymbol{y} \in \mathbb{Z}^{d}$,

$$
\left.|| \boldsymbol{y}\right|^{-1 / p}-\left.|\boldsymbol{x}|^{-1 / p}|\leq C| \boldsymbol{y}\right|^{-1 / p-1},
$$

it follows that

$$
E_{2} \leq C \sum_{s=1}^{u^{-p}} s^{-1 / p-1} M(s) \leq C \sum_{s=1}^{u^{-p}} s^{-1 / p+d-2} \leq C \max \left(1, u^{-p d+p+1}\right),
$$

since $M(s) \leq C s^{d-1}$. Combining the bounds for $E_{1}, E_{2}$, and $E_{3}$ yields Lemma 2.1.

Letting $s:=u^{-p}$ in (2.1), note that, to prove Theorem 1.1, it suffices to show that

$$
\lim _{t \rightarrow \infty} t^{1 / \alpha} \int_{1}^{\infty} \mathrm{P}[D(s)>t] \frac{1}{p} s^{-1 / p-1} \mathrm{~d} s=\beta^{1 / \alpha} .
$$

We observe that (2.3) is plausible because Lemma 2.1 suggests that $\mathrm{P}[D(s)>t]$ is close to 1 for $t \ll \beta s^{\alpha / p}$ and close to 0 for $t \gg \beta s^{\alpha / p}$, indicating that the left-hand side of (2.3) behaves like

$$
\lim _{t \rightarrow \infty} t^{1 / \alpha} \int_{(t / \beta)^{p / \alpha}}^{\infty} \frac{1}{p} s^{-1 / p-1} \mathrm{~d} s=\beta^{1 / \alpha} .
$$


To put this heuristic argument on a rigorous footing, we rewrite the integral in (2.3) as a sum of two integrals. The first integral is estimated via Bernstein's inequality and the second is handled using Poisson approximation arguments. We do this as follows.

For all $v>0$, let $m(v):=\sup \{s: \mathrm{E}[D(s)] \leq v\}$. Recalling that $\alpha:=p d-1$, from Lemma 2.1 we obtain

$$
\mathrm{E}[D(s)]=\beta s^{\alpha / p}+O\left(\max \left(1, s^{d-1-1 / p}\right)\right)=\beta s^{\alpha / p}\left(1+\max \left(O\left(s^{1 / p-d}\right), O\left(s^{-1}\right)\right)\right) .
$$

It follows, for large $v$ and $p \in(1 / d, \infty)$, that

$$
m(v)=\left(\frac{v}{(1+o(1)) \beta}\right)^{p / \alpha},
$$

where $o(1)$ tends to 0 as $v \rightarrow \infty$. Given fixed $t \geq \beta$ and $\varepsilon \in\left(0, \frac{1}{2}\right)$, define the following two integration domains:

$$
I_{1}:=\left[1, m\left(t-t^{1 / 2+\varepsilon}\right)\right), \quad I_{2}:=\left[m\left(t-t^{1 / 2+\varepsilon}\right), \infty\right) .
$$

Rewrite the left-hand side of (2.3) as

$\lim _{t \rightarrow \infty} t^{1 / \alpha} \int_{I_{1}} \mathrm{P}[D(s)>t] \frac{1}{p} s^{-1 / p-1} \mathrm{~d} s+\lim _{t \rightarrow \infty} t^{1 / \alpha} \int_{I_{2}} \mathrm{P}[D(s)>t] \frac{1}{p} s^{-1 / p-1} \mathrm{~d} s=: S_{1}+S_{2}$,

provided that both limits exist.

To prove Theorem 1.1 it suffices to show that $S_{1}=0$ and $S_{2}=\beta^{1 / \alpha}$. We first show that $S_{1}=0$. Bernstein's inequality [15, p. 12] for sums of independent, bounded random variables yields, for all $s \in I_{1}$,

$$
\mathrm{P}[D(s)>t] \leq \exp \left(\frac{-(t-\mathrm{E}[D(s)])^{2}}{2 \mathrm{E}[D(s)]+4 t / 3}\right) .
$$

Using the bounds $\inf _{s \in I_{1}}(t-\mathrm{E}[D(s)]) \geq t^{1 / 2+\varepsilon}$ and $\sup _{s \in I_{1}} \mathrm{E}[D(s)] \leq t-t^{1 / 2+\varepsilon}<t$, for all $s \in I_{1}$ we thus obtain

$$
\mathrm{P}[D(s)>t] \leq \exp \left(\frac{-\left(t^{1 / 2+\varepsilon}\right)^{2}}{10 t / 3}\right)=\exp \left(-\frac{3 t^{2 \varepsilon}}{10}\right) .
$$

It follows that

$$
S_{1} \leq \limsup _{t \rightarrow \infty} t^{1 / \alpha} \exp \left(-\frac{3 t^{2 \varepsilon}}{10}\right) \int_{1}^{\infty} \frac{1}{p} s^{-1 / p-1} \mathrm{~d} s=0 .
$$

We next show that $S_{2}=\beta^{1 / \alpha}$. By approximating $D(s)$ with a Poisson random variable we establish the following simplified expression for $S_{2}$. Here and elsewhere, $\operatorname{Po}(\lambda)$ denotes a Poisson random variable with mean $\lambda$.

Lemma 2.2. For all $p \in(1 / d, \infty)$, we have

$$
S_{2}=\lim _{t \rightarrow \infty} t^{1 / \alpha} \int_{m\left(t-t^{1 / 2+\varepsilon}\right)}^{\infty} \mathrm{P}[\operatorname{Po}(\mathrm{E}[D(s)])>t] \frac{1}{p} s^{-1 / p-1} \mathrm{~d} s .
$$

Proof. For all $\boldsymbol{y} \in \mathbb{Z}^{d}$, let $p_{\boldsymbol{y}}:=\mathrm{E}\left[1_{\left\{U_{\boldsymbol{y}} \leq|\boldsymbol{y}|^{-1 / p}\right\}}\right]=|\boldsymbol{y}|^{-1 / p}$. Letting $d_{\mathrm{TV}}$ be the total variation distance, it follows from well-known Poisson approximation bounds (e.g. Equation (1.23) 
of [3]) that

$$
d_{\mathrm{TV}}(D(s), \operatorname{Po}(\mathrm{E}[D(s)])) \leq\left(\sum_{\boldsymbol{y} \in L_{s}(\mathbf{0})} p_{\boldsymbol{y}}\right)^{-1} \sum_{\boldsymbol{y} \in L_{s}(\mathbf{0})} p_{\boldsymbol{y}}^{2} .
$$

By an analysis similar to that in the proof of Lemma 2.1 and (2.4), for $d>2 / p$ we obtain

$$
\sum_{\boldsymbol{y} \in L_{s}(\mathbf{0})} p_{\boldsymbol{y}}^{2}=\frac{p d \omega_{d}}{p d-2} s^{d-2 / p}(1+o(1)),
$$

whereas, for $1 / p<d \leq 2 / p$, we have

$$
\sum_{\boldsymbol{y} \in L_{s}(\mathbf{0})} p_{\boldsymbol{y}}^{2}=O(1)
$$

It follows from Lemma 2.1 that, for $d>2 / p$, we obtain

$$
\begin{aligned}
d_{\mathrm{TV}}(D(s), \operatorname{Po}(\mathrm{E}[D(s)])) & \leq\left(\beta s^{d-1 / p}(1+o(1))\right)^{-1} \beta\left(\frac{p d-1}{p d-2}\right) s^{d-2 / p}(1+o(1)) \\
& =O\left(s^{-1 / p}\right),
\end{aligned}
$$

whereas, for $1 / p<d \leq 2 / p$, we have

$$
d_{\mathrm{TV}}(D(s), \operatorname{Po}(\mathrm{E}[D(s)]))=O\left(s^{-d+1 / p}\right) .
$$

Letting

$$
e(s, t):=\mathrm{P}[D(s)>t]-\mathrm{P}[\mathrm{Po}(\mathrm{E}[D(s)])>t],
$$

it follows that, uniformly in $t \in(0, \infty)$, we have $|e(s, t)|=O\left(s^{-\xi}\right)$, where $\xi=1 / p$ for $d>2 / p$ and $\xi=d-1 / p$ for $1 / p<d \leq 2 / p$. We now rewrite $S_{2}$ as

$$
S_{2}=\lim _{t \rightarrow \infty} t^{1 / \alpha} \int_{m\left(t-t^{1 / 2+\varepsilon}\right)}^{\infty}(\mathrm{P}[\mathrm{Po}(\mathrm{E}[D(s)])>t]+e(s, t)) \frac{1}{p} s^{-1 / p-1} \mathrm{~d} s
$$

and show that the term containing $e(s, t)$ is negligible.

Recall that

$$
m\left(t-t^{1 / 2+\varepsilon}\right)=\left(\frac{t-t^{1 / 2+\varepsilon}}{(1+o(1)) \beta}\right)^{p / \alpha},
$$

where, here and in the remainder of this section, $o(1)$ tends to 0 as $t \rightarrow \infty$. It follows that

$$
\int_{m\left(t-t^{1 / 2+\varepsilon}\right)}^{\infty} e(s, t) s^{-1 / p-1} \mathrm{~d} s=O\left(\int_{m\left(t-t^{1 / 2+\varepsilon}\right)}^{\infty} s^{-\xi-1 / p-1} \mathrm{~d} s\right)=O\left(t^{-p / \alpha(\xi+1 / p)}\right)
$$

and, therefore, that

$$
\lim _{t \rightarrow \infty} t^{1 / \alpha} \int_{m\left(t-t^{1 / 2+\varepsilon}\right)}^{\infty} e(s, t) s^{-1 / p-1} \mathrm{~d} s=0 .
$$

We thus obtain Lemma 2.2.

It is now straightforward to show that $S_{2}=\beta^{1 / \alpha}$. Letting $z:=\beta s^{d-1 / p} / t$, whence $s=$ $(t z / \beta)^{p / \alpha}$ and $\mathrm{E}[D(s)]=t z\left(1+O\left((t z)^{-\rho}\right)\right)$ with $\rho:=\rho(p, d)>0$, we obtain, via Lemma 2.2 ,

$$
S_{2}=\lim _{t \rightarrow \infty} \frac{\beta^{1 / \alpha}}{\alpha} \int_{1+o(1)}^{\infty} \mathrm{P}\left[\operatorname{Po}\left(t z\left(1+O\left((t z)^{-\rho}\right)\right)\right)>t\right] z^{-1 / \alpha-1} \mathrm{~d} z
$$


The integrability of the integrand on $[1+o(1), \infty)$ gives, for all $\gamma>0$,

$$
S_{2}=\lim _{t \rightarrow \infty} \frac{\beta^{1 / \alpha}}{\alpha} \int_{1+\gamma}^{\infty} \mathrm{P}\left[\operatorname{Po}\left(t z\left(1+O\left((t z)^{-\rho}\right)\right)\right)>t\right] z^{-1 / \alpha-1} \mathrm{~d} z+\gamma \cdot O(1) .
$$

For all $z \in[1+\gamma, \infty)$, we have $\mathrm{P}\left[\operatorname{Po}\left(t z\left(1+O\left((t z)^{-\rho}\right)\right)\right)>t\right] \rightarrow 1$ as $t \rightarrow \infty$. The dominated convergence theorem yields

$$
S_{2}=\frac{\beta^{1 / \alpha}}{\alpha} \int_{1}^{\infty} z^{-1 / \alpha-1} \mathrm{~d} z+\gamma O(1)=\beta^{1 / \alpha}+\gamma \cdot O(1) .
$$

Now let $\gamma \rightarrow 0$ to obtain $S_{2}=\beta^{1 / \alpha}$, as desired.

\section{Proof of Theorem 1.2}

We prove Theorem 1.2 by showing, for all $\boldsymbol{x} \in \mathbb{Z}^{d}$, the existence of an event $E:=E(\boldsymbol{x}) \subset \Omega$, with $\mathrm{P}[E]=1-o(1)$, such that on $E$ there is a path $\pi$ consisting of $N$ edges in $G_{p}\left(\mathbb{Z}^{d}\right)$ joining 0 to $\boldsymbol{x}$, where $N \leq 4(2+\log \log |\boldsymbol{x}|)$. Here and in the sequel, $o(1)$ denotes a quantity tending to 0 as $|\boldsymbol{x}| \rightarrow \infty$.

Constructing the path $\pi$ would be easy if the balls of influence at $\mathbf{0}$ and $\boldsymbol{x}$ both had radius at least $|\boldsymbol{x}|$, for then $\pi$ would consist merely of the single edge $(\mathbf{0}, \boldsymbol{x})$. In general, the balls of influence at $\mathbf{0}$ and $\boldsymbol{x}$ have much smaller radii and the path $\pi$ thus needs to join a sequence of balls such that consecutive balls contain each other's centers.

The heart of the proof will consist of constructing a sequence of nodes of cardinality roughly $2 \log \log |\boldsymbol{x}|$ with these properties: the first node, $\mathbf{0}^{\prime}$, is at distance at most $\frac{1}{2} \log \log |\boldsymbol{x}|$ from $\mathbf{0}$; the last node, $\boldsymbol{x}^{\prime}$, is at distance at most $\frac{1}{2} \log \log |\boldsymbol{x}|$ from $\boldsymbol{x}$; and the edges defined by consecutive nodes are in $G_{p}$, i.e. the balls of influence at consecutive nodes contain each other's centers. Since $\mathbf{0}$ and $\mathbf{0}^{\prime}$ can be joined with a path of at most $\log \log |\boldsymbol{x}|$ edges, and likewise for $\boldsymbol{x}$ and $\boldsymbol{x}^{\prime}$, we can obtain a path $\pi$ consisting of roughly $4 \log \log |\boldsymbol{x}|$ edges. The construction of this sequence of nodes depends critically on an intermediate node, denoted here by $\boldsymbol{P}_{0}$, that has an unusually large ball of influence. Before defining $\mathbf{0}^{\prime}, \boldsymbol{P}_{0}$, and $\boldsymbol{x}^{\prime}$, we need some terminology.

For all $\boldsymbol{x} \in \mathbb{R}^{d}$ and $r>0$, let $L_{r}^{+}(\boldsymbol{x})$ and $L_{r}^{-}(\boldsymbol{x})$ denote the lattice points in the upper and lower hemispheres of radius $r$ centered at $\boldsymbol{x}$. That is, $L_{r}^{+}(\boldsymbol{x}):=B_{r}(\boldsymbol{x}) \cap\left(\mathbb{Z}^{d-1} \times \mathbb{Z}^{+}\right)$and, similarly, $L_{r}^{-}(\boldsymbol{x}):=B_{r}(\boldsymbol{x}) \cap\left(\mathbb{Z}^{d-1} \times \mathbb{Z}^{-}\right)$. Here $\mathbb{Z}^{+}:=\{1,2, \ldots\}$ and $\mathbb{Z}^{-}:=\{-1,-2, \ldots\}$.

\subsection{Definition of $0^{\prime}, P_{0}$, and $x^{\prime}$}

Throughout, we appeal to the following elementary fact. Recall that $\log s$ is short for $\log _{p d} s$.

Lemma 3.1. Let $U_{1}, \ldots, U_{n}$ be independent and identically uniformly distributed on $[0,1]$. Then, for all $n>p d$, we have

$$
\min _{i \leq n} U_{i} \leq \frac{K \log n}{n}
$$

with probability at least $1-n^{-K}$.

In the sequel, we fix $K$ to be large, with a value to be determined later.

3.1.1. Definition of $\mathbf{0}^{\prime}$. Let $E_{\mathbf{0}}:=E_{\mathbf{0}}(\boldsymbol{x})$ be the event that there is a node $z \in L_{(1 / 2) \log \log |\boldsymbol{x}|}^{-}(\mathbf{0})$ such that

$$
U_{z} \leq \frac{K \log (\log \log |x|)^{d}}{(\log \log |x|)^{d}}
$$


Clearly, $E_{\mathbf{0}}$ depends only on $U_{z}, z \in L_{(1 / 2) \log \log |x|}^{-}(\mathbf{0})$. In case more than one node in $L_{(1 / 2) \log \log |x|}^{-}(\mathbf{0})$ satisfies the last bound, we choose $z$ to be that node with smallest lexicographical order.

By Lemma 3.1, $\mathrm{P}\left[E_{\mathbf{0}}\right] \geq 1-C(\log \log |\boldsymbol{x}|)^{-d K}$. Given $E_{\mathbf{0}}$ we let $\mathbf{0}^{\prime}:=\boldsymbol{z}$. Note that $\mathbf{0}^{\prime}$ is random and that, since $p d>1$, for all large $|\boldsymbol{x}|$ we have

$$
U_{\mathbf{0}^{\prime}}^{-p} \geq 2 \log \log |\boldsymbol{x}| \text {. }
$$

Inequality (3.1) will be important in the sequel. For now note that, since $G_{p}\left(\mathbb{Z}^{d}\right)$ connects adjacent lattice points, it follows that $d_{p}(\boldsymbol{y}, \boldsymbol{x}) \leq 2|\boldsymbol{y}-\boldsymbol{x}|$ for all $\boldsymbol{x}, \boldsymbol{y} \in \mathbb{Z}^{d}$, i.e. that

$$
d_{p}\left(\mathbf{0}, \mathbf{0}^{\prime}\right) \leq \log \log |\boldsymbol{x}| \text {. }
$$

3.1.2. Definition of $\boldsymbol{x}^{\prime}$. Similarly, given $\boldsymbol{x}$, with probability at least $1-C(\log \log |\boldsymbol{x}|)^{-d K}$ there is an event $E_{\boldsymbol{x}}$ such that there is a node $\boldsymbol{x}^{\prime} \in L_{(1 / 2) \log \log |\boldsymbol{x}|}^{-}(\boldsymbol{x})$ on $E_{\boldsymbol{x}}$ with weight

$$
U_{\boldsymbol{x}^{\prime}}^{-p} \geq 2 \log \log |\boldsymbol{x}| \text {. }
$$

Clearly $d_{p}\left(\boldsymbol{x}, \boldsymbol{x}^{\prime}\right) \leq \log \log |\boldsymbol{x}|$ and $E_{\boldsymbol{x}}$ depends only on $U_{z}, z \in L_{(1 / 2) \log \log |\boldsymbol{x}|}^{-}(\boldsymbol{x})$.

3.1.3. Definition of $\boldsymbol{P}_{0}$. Assume without loss of generality that the components of $\boldsymbol{x}$ have even parity, meaning that $\boldsymbol{x} / 2 \in \mathbb{Z}^{d}$. Consider the event, $E_{\boldsymbol{x} / 2}$, that there is a node $\boldsymbol{P}_{0} \in L_{|\boldsymbol{x}| / 10}(\boldsymbol{x} / 2)$ with

$$
U_{\boldsymbol{P}_{0}} \leq \frac{K \log (|\boldsymbol{x}|)^{d}}{|\boldsymbol{x}|^{d}} .
$$

Lemma 3.1 implies that $\mathrm{P}\left[E_{\boldsymbol{x} / 2}\right] \geq 1-C\left(|\boldsymbol{x}|^{-d K}\right)$. We note that, for large $|\boldsymbol{x}|$,

$$
U_{\boldsymbol{P}_{0}}^{-p} \geq 2|\boldsymbol{x}|
$$

since $p d>1$.

\subsection{Construction of the path $\pi$ via $0^{\prime}, P_{0}$, and $x^{\prime}$}

It will suffice to show that there is an event $E:=E(\boldsymbol{x})$, with $\mathrm{P}[E(\boldsymbol{x})]=1-o(1)$, such that on $E$ there are two paths, each having at most $2+2\lceil\log \log |\boldsymbol{x}|\rceil$ edges, with one path joining $\boldsymbol{P}_{0}$ to $\mathbf{0}$ and the other joining $\boldsymbol{P}_{0}$ to $\boldsymbol{x}$. It will be enough to show the existence of a path between $\boldsymbol{P}_{0}$ and $\mathbf{0}$, for the method can be repeated verbatim to yield the path between $\boldsymbol{P}_{0}$ and $\boldsymbol{x}$. We first introduce some additional terminology.

We abbreviate our notation by letting $b:=p d$. Note that $b>1$ by assumption. Fix $\varepsilon \in(0,1)$ and $\boldsymbol{x} \in \mathbb{Z}^{d}$ with $|\boldsymbol{x}|$ large. For all $j=1,2, \ldots$, let

$$
r_{j}:=r_{j}(\boldsymbol{x}, \varepsilon):=|\boldsymbol{x}|^{b^{-j(1-\varepsilon)}}
$$

and note that $r_{j} \downarrow 1$ and $1<r_{j}<|\boldsymbol{x}|$ for all $j=1,2, \ldots$ We record an elementary fact.

Lemma 3.2. $r_{j+1}=r_{j}^{\beta(p, d, \varepsilon)}$, where $\beta(p, d, \varepsilon):=b^{-1+\varepsilon}$.

For all $j=1,2, \ldots$, consider the following disjoint 'semi-annular' regions of lattice points:

$$
A_{j}:=\left[\left(L_{r_{j}}^{+}\left(\mathbf{0}^{\prime}\right)-L_{r_{j+1}}^{+}\left(\mathbf{0}^{\prime}\right)\right) \backslash L_{|\boldsymbol{x}| / 10}^{+}(\boldsymbol{x} / 2)\right] .
$$

The construction of the path joining $\boldsymbol{P}_{0}$ to $\mathbf{0}$ is facilitated by the following four lemmas. The first three lemmas show that, for all $j, 1 \leq j \leq\lceil\log \log |\boldsymbol{x}|\rceil+1$, there are points $\boldsymbol{P}_{j} \in A_{j}$ such that $\left(\boldsymbol{P}_{j}, \boldsymbol{P}_{j-1}\right)$ and $\left(\boldsymbol{P}_{\lceil\log \log |\boldsymbol{x}|\rceil+1}, \mathbf{0}^{\prime}\right)$ belong to $G_{p}\left(\mathbb{Z}^{d}\right)$. The fourth lemma shows that this happens on an event with probability $1-o(1)$. By consecutively linking $\boldsymbol{P}_{j}, 0 \leq j \leq\lceil\log \log |\boldsymbol{x}|\rceil+1$, and $\mathbf{0}^{\prime}$, we construct a path joining $\boldsymbol{P}_{0}$ to $\mathbf{0}^{\prime}$ with $\lceil\log \log |\boldsymbol{x}|\rceil+2$ 
edges. Since $\mathbf{0}^{\prime}$ is within $\frac{1}{2} \log \log |\boldsymbol{x}|$ of $\mathbf{0}$, we need at most $\lceil\log \log |\boldsymbol{x}|\rceil$ edges to join $\mathbf{0}^{\prime}$ to $\mathbf{0}$ (recall (3.2)). This gives a path joining $\boldsymbol{P}_{0}$ to $\mathbf{0}$ with at most $2\lceil\log \log |\boldsymbol{x}|\rceil+2$ edges. Since $2+2\lceil\log \log |\boldsymbol{x}|\rceil \leq 4+2 \log \log |\boldsymbol{x}|$, we obtain Theorem 1.2, as desired. We now turn to our four lemmas.

Lemma 3.3. There exists an event $E_{1}$, with $\mathrm{P}\left[E_{1}\right]=1-O\left(r_{1}^{-d K}\right)$, such that on $E_{1}$ there is a node $\boldsymbol{P}_{1} \in A_{1}$ which is linked to $\boldsymbol{P}_{0}$, i.e. the edge $\left(\boldsymbol{P}_{0}, \boldsymbol{P}_{1}\right)$ is in $G_{p}\left(\mathbb{Z}^{d}\right)$.

Proof. The number of lattice points in $A_{1}$ is $\Theta\left(|\boldsymbol{x}|^{d b^{-1+\varepsilon}}\right)$, i.e. there is a constant $C>0$ such that the number of lattice points is bounded from above by $C\left(|\boldsymbol{x}|^{d b^{-1+\varepsilon}}\right)$ and bounded from below by $C^{-1}\left(|\boldsymbol{x}|^{d b^{-1+\varepsilon}}\right)$. Lemma 3.1 implies that there is an event $E_{1}$, depending only on $\left\{U_{z}\right\}_{z \in A_{1}}$ and with

$$
\mathrm{P}\left[E_{1}\right]=1-O\left(|\boldsymbol{x}|^{-d K b^{-1+\varepsilon}}\right),
$$

such that, for large $|\boldsymbol{x}|, E_{1}$ implies the existence of $\boldsymbol{P}_{1} \in A_{1}$ with

$$
U_{\boldsymbol{P}_{1}} \leq \frac{K \log \left(|\boldsymbol{x}|^{d b^{-1+\varepsilon}}\right)}{|\boldsymbol{x}|^{d b^{-1+\varepsilon}}}
$$

Again, if there is more than one node in $A_{1}$ satisfying this inequality, we choose the one with smallest lexicographical order. Since $b:=p d$ it follows for large $|\boldsymbol{x}|$ that $\boldsymbol{P}_{1}$ has weight

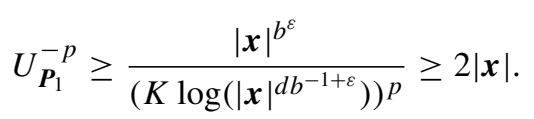

We now show that $\boldsymbol{P}_{1}$ is linked to $\boldsymbol{P}_{0}$. It suffices to show that

$$
\left|\boldsymbol{P}_{0}-\boldsymbol{P}_{1}\right| \leq \min \left(U_{\boldsymbol{P}_{0}}^{-p}, U_{\boldsymbol{P}_{1}}^{-p}\right) .
$$

However, $\left|\boldsymbol{P}_{0}-\boldsymbol{P}_{1}\right| \leq\left|\boldsymbol{P}_{0}\right|+\left|\boldsymbol{P}_{1}\right| \leq 2|\boldsymbol{x}|$, so Lemma 3.3 follows from (3.3) and (3.4).

Given $\boldsymbol{x}$, let $m:=m(\boldsymbol{x})$ denote the largest integer such that $r_{m} \geq \log \log |\boldsymbol{x}| ; m$ is well defined since $r_{j} \downarrow 1$. If $t:=[1 /(1-\varepsilon)] \log \log |\boldsymbol{x}|$ then

$$
|\boldsymbol{x}|^{b^{-t(1-\varepsilon)}}=|\boldsymbol{x}|^{1 / \log |\boldsymbol{x}|}=b,
$$

showing that $m$ is bounded by $t$. The next lemma extends the arguments of Lemma 3.3 and builds a path of $m$ edges from $\boldsymbol{P}_{0}$ to a node $\boldsymbol{P}_{m} \in A_{m}$.

Lemma 3.4. For all $j, 1 \leq j \leq m$, there is an event $E_{j}$, depending only on $\left\{U_{z}\right\}_{z \in A_{j}}$, such that

(i) $\mathrm{P}\left[E_{j}\right]=1-O\left(r_{j}^{-d K}\right)$, and

(ii) on each $E_{j}$ there is a node $\boldsymbol{P}_{j} \in A_{j}$ such that, on $E_{j-1} \cap E_{j}$, the edge $\left(\boldsymbol{P}_{j-1}, \boldsymbol{P}_{j}\right)$ is in $G_{p}$.

Proof. Since card $\left\{A_{j}\right\}=\Theta\left(r_{j}^{d}\right)$, Lemma 3.1 implies that for large $|\boldsymbol{x}|$ there is an event $E_{j}$, with $\mathrm{P}\left[E_{j}\right]=1-O\left(r_{j}^{-d K}\right)$, which depends only on $\left\{U_{z}\right\}_{z \in A_{j}}$ and which implies the existence of a $\boldsymbol{P}_{j} \in A_{j}$ satisfying

$$
U_{\boldsymbol{P}_{j}} \leq \frac{K \log \left(r_{j}^{d}\right)}{r_{j}^{d}}=: W_{j}
$$


It remains to show that

$$
\left|\boldsymbol{P}_{j}-\boldsymbol{P}_{j-1}\right| \leq \min \left(U_{\boldsymbol{P}_{j}}^{-p}, U_{\boldsymbol{P}_{j-1}}^{-p}\right)
$$

for all $j, 1 \leq j \leq m$. Lemma 3.3 shows (3.5) for $j=1$. The maximal distance between points in $A_{j}$ and $A_{j-1}$ is at most twice $r_{j-1}$, i.e. $\left|\boldsymbol{P}_{j}-\boldsymbol{P}_{j-1}\right| \leq 2 r_{j-1}$. It thus suffices to show that

$$
2 r_{j-1} \leq \min \left(W_{j}^{-p}, W_{j-1}^{-p}\right)=W_{j}^{-p},
$$

which holds since $W_{j-1}^{-p} \geq W_{j}^{-p}$ for all $j, 1 \leq j \leq m$.

However, by Lemma 3.2,

$$
W_{j}^{-p}=\frac{r_{j}^{p d}}{\left(K d \log r_{j}\right)^{p}}=\frac{\left(\left(r_{j-1}\right)^{b^{-1+\varepsilon}}\right)^{p d}}{\left(K d b^{-1+\varepsilon} \log \left(r_{j-1}\right)\right)^{p}} .
$$

Thus, for all $j, 1 \leq j \leq m$,

$$
\frac{W_{j}^{-p}}{r_{j-1}}=\frac{\left(r_{j-1}\right)^{b^{\varepsilon}-1}}{\left(K d b^{-1+\varepsilon} \log \left(r_{j-1}\right)\right)^{p}} \geq \frac{\left(r_{m}\right)^{b^{\varepsilon}-1}}{\left(K d b^{-1+\varepsilon} \log \left(r_{m}\right)\right)^{p}},
$$

since the $r_{j}$ are decreasing. By definition of $r_{m}$ and since $b^{\varepsilon}-1>0$, the last ratio clearly exceeds 2 for large $|\boldsymbol{x}|$, showing (3.6) and completing the proof of Lemma 3.4.

The next lemma shows that we may link $\boldsymbol{P}_{m}$ and $\mathbf{0}^{\prime}$ via a node $\boldsymbol{P}_{m+1} \in A_{m+1}$. Combined with Lemmas 3.2 and 3.3, this builds a path between $\boldsymbol{P}_{0}$ and $\mathbf{0}^{\prime}$ which contains $m+2$ edges.

Lemma 3.5. There is an event $E_{m+1}$, depending only on $\left\{U_{z}\right\}_{z \in A_{m+1}}$, such that $\mathrm{P}\left[E_{m+1}\right]=$ $1-O\left(r_{m+1}^{-d K}\right)$, and on $E_{\mathbf{0}} \cap E_{m} \cap E_{m+1}$ there is a point $\boldsymbol{P}_{m+1} \in A_{m+1}$ such that the edges $\left(\boldsymbol{P}_{m}, \boldsymbol{P}_{m+1}\right)$ and $\left(\boldsymbol{P}_{m+1}, \mathbf{0}^{\prime}\right)$ both belong to $G_{p}\left(\mathbb{Z}^{d}\right)$.

Proof. First, by definition of $m$ and by Lemma 3.2 we have

$$
(\log \log |\boldsymbol{x}|)^{\beta} \leq r_{m}^{\beta}=r_{m+1}<\log \log |\boldsymbol{x}| .
$$

By Lemma 3.1, for large $|\boldsymbol{x}|$ there is an event $E_{m+1}$, with $\mathrm{P}\left[E_{m+1}\right]=1-O\left(r_{m+1}^{-d K}\right)$, which depends only on $\left\{U_{z}\right\}_{z \in A_{m+1}}$ and which implies the existence of a point $\boldsymbol{P}_{m+1} \in A_{m+1}$ with

$$
U_{\boldsymbol{P}_{m+1}} \leq \frac{K \log \left(r_{m+1}^{d}\right)}{r_{m+1}^{d}} \leq \frac{K \log (\log \log |\boldsymbol{x}|)^{d}}{(\log \log |\boldsymbol{x}|)^{\beta d}} \leq \frac{K \log (\log \log |\boldsymbol{x}|)^{d}}{(\log \log |\boldsymbol{x}|)^{(p d)^{\varepsilon} / p}}
$$

since $\beta d=(p d)^{\varepsilon} / p$. Since $(p d)^{\varepsilon}>1$, it follows that, for large $|\boldsymbol{x}|$, on $E_{m+1}$ we have

$$
U_{P_{m+1}}^{-p} \geq 2 \log \log |\boldsymbol{x}| \text {. }
$$

Following the arguments in the proof of Lemma 3.4 (with $j$ equal to $m+1$ there), we find that, on $E_{m} \cap E_{m+1},\left(\boldsymbol{P}_{m}, \boldsymbol{P}_{m+1}\right)$ is an edge in $G_{p}\left(\mathbb{Z}^{d}\right)$. Furthermore, on $E_{\mathbf{0}} \cap E_{m} \cap E_{m+1}$, the edge $\left(\boldsymbol{P}_{m+1}, \mathbf{0}^{\prime}\right)$ belongs to $G_{p}\left(\mathbb{Z}^{d}\right)$ if and only if

$$
\left|\mathbf{0}^{\prime}-\boldsymbol{P}_{m+1}\right| \leq \min \left(U_{\mathbf{0}^{\prime}}^{-p}, U_{\boldsymbol{P}_{m+1}}^{-p}\right) .
$$

However,

$$
\left|\boldsymbol{0}^{\prime}-\boldsymbol{P}_{m+1}\right| \leq\left|\mathbf{0}^{\prime}-\mathbf{0}\right|+\left|\mathbf{0}-\boldsymbol{P}_{m+1}\right| \leq \log \log |\boldsymbol{x}|+r_{m+1} \leq 2 \log \log |\boldsymbol{x}|,
$$

showing that (3.8) follows, using (3.7) and (3.1). 
The last lemma completes the proof of Theorem 1.2.

Lemma 3.6. For all $\boldsymbol{x} \in \mathbb{Z}^{d}$, there is an event $E(\boldsymbol{x})$, with $\mathrm{P}[E(\boldsymbol{x})]=1-o(1)$, such that on $E(\boldsymbol{x})$ there exists a path joining $\boldsymbol{P}_{0}$ to $\mathbf{0}$ with $4+2 \log \log |\boldsymbol{x}|$ edges.

Proof. Let $E(\boldsymbol{x}):=E_{\mathbf{0}} \cap E_{\boldsymbol{x} / 2} \cap\left(\bigcap_{j=1}^{m+1} E_{j}\right)$. On $E(\boldsymbol{x})$ we have shown that there is a path, $\pi$, joining $\boldsymbol{P}_{0}$ to $\mathbf{0}$ via the successive nodes $\boldsymbol{P}_{1}, \boldsymbol{P}_{2}, \ldots, \boldsymbol{P}_{m}, \boldsymbol{P}_{m+1}, \mathbf{0}^{\prime}, \mathbf{0}$. The number of edges in $\pi$ is bounded by $m+2+\lceil\log \log |\boldsymbol{x}|\rceil$, where $\lceil\log \log |\boldsymbol{x}|\rceil$ denotes an upper bound on the number of edges between $\mathbf{0}^{\prime}$ and $\mathbf{0}$. Since $\varepsilon$ is arbitrary in the definition of $t$, it follows that $m \leq\lceil\log \log |\boldsymbol{x}|\rceil$. Thus, $\operatorname{card}\{\pi\} \leq 4+2 \log \log |\boldsymbol{x}|$.

Finally, we show that $\mathrm{P}[E(\boldsymbol{x})]=1-o(1)$. For all $j, 1 \leq j \leq m+1, E_{j}$ depends only on $\left\{U_{z}\right\}_{z \in A_{j}}$ and, since the $A_{j}$ are disjoint, the $\left\{E_{j}\right\}_{1 \leq j \leq m+1}$ are independent. Clearly, since

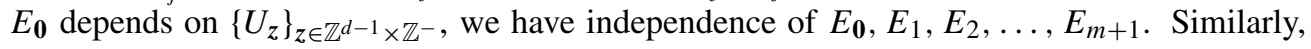
$E_{\boldsymbol{x} / 2}, E_{\mathbf{0}}, E_{1}, E_{2}, \ldots, E_{m+1}$ are independent.

By independence, we have

$$
\mathrm{P}[E(\boldsymbol{x})]=\mathrm{P}\left[\bigcap_{j=1}^{m+1} E_{j}\right] \mathrm{P}\left[E_{\mathbf{0}}\right] \mathrm{P}\left[E_{\boldsymbol{x}}\right] \mathrm{P}\left[E_{\boldsymbol{x} / 2}\right]=(1-o(1))^{3} \prod_{j=1}^{m+1} \mathrm{P}\left[E_{j}\right] .
$$

Now, $m$ is bounded by $C \log \log |\boldsymbol{x}|$ and the definition of $r_{m}$ shows, for large $K$, that $m r_{m+1}^{-d K} \rightarrow 0$ as $|\boldsymbol{x}| \rightarrow \infty$. Since $1-2 s \leq \exp (-s) \leq 1-s / 2$ for small, positive $s$, it follows that

$$
\begin{aligned}
\prod_{j=1}^{m+1} \mathrm{P}\left[E_{j}\right] & =\prod_{j=1}^{m+1}\left(1-O\left(r_{j}^{-d K}\right)\right) \\
& \geq \exp \left(-C \sum_{j=1}^{m+1} r_{j}^{-d K}\right) \\
& \geq 1-C \sum_{j=1}^{m+1} r_{j}^{-d K} \\
& \geq 1-C \sum_{j=1}^{m+1} r_{m+1}^{-d K} .
\end{aligned}
$$

This yields $\mathrm{P}[E(\boldsymbol{x})]=1-o(1)$, as desired, completing the proof of Lemma 3.6.

\section{Proof of Theorem 1.3}

Assume without loss of generality that $n$ has even parity. Partition $[0, n]^{d} \cap \mathbb{Z}^{d}$ into $Q_{1}:=$ $\left[0, \frac{1}{2} n\right] \times[0, n]^{d-1} \cap \mathbb{Z}^{d}$ and $Q_{2}:=\left(\frac{1}{2} n, n\right] \times[0, n]^{d-1} \cap \mathbb{Z}^{d}$. For all $k=0,1,2, \ldots, n / 2$, write $Q_{1, k}:=\{n / 2-k\} \times[0, n]^{d-1} \cap \mathbb{Z}^{d}$ and note that $Q_{1}=\bigcup_{k=0}^{n / 2} Q_{1, k}$.

The number of nodes in $Q_{1}$ whose balls of influence have nonempty intersection with $Q_{2}$ is

$$
N:=\sum_{k=0}^{n} \sum_{i \in Q_{1, k}} 1_{\left\{U_{i}^{-p} \geq k+1\right\}}
$$


If we remove these $N$ nodes from $Q_{1}$ then $G_{p}\left(Q_{1}\right)$ and $G_{p}\left(Q_{2}\right)$ are disconnected, i.e. the graphs have no edges joining them. Moreover, as the number of nodes in $Q_{1, k}$ equals $n^{d-1}$, we obtain

$$
\mathrm{E}[N]=\sum_{k=0}^{n} n^{d-1} \mathrm{P}\left[U_{\mathbf{0}}^{-p} \geq k+1\right]=n^{d-1} \sum_{k=0}^{n}(k+1)^{-1 / p} \leq C n^{d-1}\left[n^{1-1 / p} \vee 1\right],
$$

which is exactly the desired upper bound.

\section{Acknowledgements}

I thank an anonymous referee for helpful comments and for pointing out an error in the original proof of Theorem 1.1. I also thank Mathew Penrose for helpful conversations on power-law graphs.

\section{References}

[1] Albert, R. and Barabási, A.-L. (2002). Statistical mechanics of complex networks. Rev. Modern Physics 74, 47-97.

[2] Barabási, A.-L. (2002). Linked: The New Science of Networks. Perseus, Cambridge, MA.

[3] Barbour, A. D., Holst, L. and Janson, S. (1992). Poisson Approximation. Oxford University Press.

[4] Benjamini, I., Kesten, H., Peres, Y. and Schramm, O. (2004). The geometry of the uniform spanning forests: transitions in dimensions 4, 8, 12, ... Ann. Math. 160, 465-491.

[5] Berger, N. (2004). A lower bound for the chemical distance in sparse long-range percolation models. Preprint 0409021, Department of Mathematics, University of California, Davis. Available at http://front.math. ucdavis.edu.

[6] Berger, N. et al. (2003). Degree distribution of the FKP network model. In Automata, Languages and Programming (Lecture Notes Comput. Sci. 2719), Springer, Heidelberg, pp. 725-738.

[7] BISKUP, M. (2004). Graph diameter in long-range percolation. Submitted.

[8] Biskup, M. (2004). On scaling of the chemical distance in long-range percolation models. Ann. Prob. 32, 2938-2977.

[9] Bollabás, B. and Riordan, O. M. (2003). The diameter of a scale-free random graph. Combinatorica 24, 5-34.

[10] Chung, F. And Lu, L. (2002). The average distance in a random graph with given expected degrees. Proc. Nat. Acad. Sci. USA 99, 15879-15882.

[11] Chung, F. AND Lu, L. (2003). The average distance in a random graph with given expected degrees. Internet Math. 1, 91-113. (This is an expanded version of [10].)

[12] Cohen, R. and Havlin, S. (2003). Scale-free networks are ultrasmall. Phys. Rev. Lett. 90, 058701.

[13] Coppersmith, D., Gamarnik, D. And Sviridenko, M. (2002). The diameter of a long-range percolation graph. Random Structures Algorithms 21, 1-13.

[14] Dorogovtsev, S. N. And Mendes, J. F. F. (2003). Evolution of Networks: From Biological Nets to the Internet and World Wide Web. Oxford University Press.

[15] Dudley, R. M. (1999). Uniform Central Limit Theorems. Cambridge University Press.

[16] Fabrikant, A., Koutsoupias, E. and Papadimitriou, C. H. (2002). Heuristically optimized trade-offs: a new paradigm for power laws in the internet. In Automata, Languages and Programming (Lecture Notes Comput. Sci. 2380), Springer, Berlin, pp. 110-122.

[17] Franceschetti, M. and Meester, R. (2004). Navigation in small world networks, a scale-free continuum model. Tech. Rep. UCB/ERL M03/33, EECS Department, University of California, Berkeley.

[18] Hermann, C., Barthélemy, M. and Provero, P. (2003). Connectivity distribution of spatial networks. Phys. Rev. $E$ 68, 026128.

[19] Kleinberg, J. M. (2000). Navigation in the small world. Nature 406, 845.

[20] Meester, R. and Roy, R. (1996). Continuum Percolation (Camb. Tracts Math. 119). Cambridge University Press.

[21] Newman, M. E. J. (2003). The structure and function of complex networks. SIAM Rev. 45, 167-256.

[22] Penrose, M. D. (2001). A spatial central limit theorem with applications to percolation, epidemics and Boolean models. Ann. Prob. 29, 1515-1546.

[23] Penrose, M. D. (2003). Random Geometric Graphs. Clarendon Press, Oxford.

[24] Watts, D. J. (1999). Small Worlds. Princeton University Press.

[25] Watts, D. J. and Strogatz, S. H. (1998). Collective dynamics of 'small world' networks. Nature 393, 440-442. 